\title{
Identification of connection in spacecraft images and classifying them for Weather Monitoring
}

\author{
Ramya S Gowda \\ Lecturer, Sikkim Manipal University, Bangalore-560008 \\ raashiramya@gmail.com
}

\begin{abstract}
In this paper we discuss on a system where in automatically the images collected from the spacecraft are identified for the connected images. the satellite images taken consist of not only basic shapes with regular size and shape but also the interconnected irregular shapes which is identified as network. Now the images that are automatically classified are either normal weather patterns or adverse weather patterns developed. An early warning is raised based on the images classification if the tendency of adverse weather system is about to be formed. In order to do this the strong knowledge of angular movement of the clouds, the knowledge about clouds, image processing and Neural Networks techniques are essential. The knowledge about clouds helps in analyzing the image data based on which decision can be taken. The image processing knowledge helps in classifying the images and the Neural Networks techniques helps in processing the interconnected images of the selected region.
\end{abstract}

Since the cloud formation is arbitrary in nature, identification of clouds as segments in the image has to use the techniques of Neural Networks.

\section{KEYWORDS}

Image processing, Pattern recognition, Neural Networks, Satellite Images, reference image, successive image, center, proximity, clockwise, and anticlockwise direction.

\section{INTRODUCTION}

Due to the attraction of the sun, Moon or because of the solar radiation pressure, the spacecraft orbits experience disturbances. These deviations are determined using ranging and tracking data, and are corrected periodically once in 80 days. This process is called station-=keeping man ever.

Weather is a state of the atmosphere at a particular time and place. The element of weather includes temperature, humidity, cloudiness, and perception. When we talk about weather, there will be perception that the word climate and weather are same. But in reality there is difference. Climate is what we expect, weather is what we get. Climate is about long-term records, trends and averages; weather is the day-to-day experience. If weather is the watch then the climate is the calendar.

1.1 Satellite images: INSAT-2E, the last of INSAT-2 series of satellites built by ISRO is a multipurpose satellite for telecommunications, television broad casting and meteorological services including disaster-warning services. Very High Resolution Radiometer (VHRR) with $2 \mathrm{~km}$ resolution in visible band and $8 \mathrm{~km}$ resolution in infrared and water vapour band. Charge Coupled Device (CCD) camera operating in visible, near infrared and short wave infrared band with $1 \mathrm{~km}$ resolution. The weather monitoring using satellite imageries play an 
important role in National Development. INDIAN NATIONAL SATELLITE SYSTEMS, INSATS provide continuous monitoring of weather pattern through a series of satellites located over Indian Ocean region. The imageries are collected from the satellites and the data is processed by India Meteorological Department at New Delhi and necessary predictions are made for use by difference agencies. INSAT spacecrafts are capable of providing the imageries at every half hour intervals in case of any urgent need. Normally about 15 times in a day these imageries are obtained from one satellite by India Meteorological Department. In case of any adverse weather system development, like cyclone, additional imageries are taken at more frequent intervals.

1.2 Relevance of DIP and PR: Digital Image Processing encompasses processes whose inputs and outputs are images and, in addition extract attributes from images, up to and including the recognition of individual objects. The processes of acquiring an image of the interested region, pre-processing that image, extracting the region of interest ( segmenting), describing the region in suitable form for computer processing and recognizing those regions in the images are in the scope of Digital Image Processing. Making sense of the content of the page may be viewed as being in the domain of automated analysis and classification of images. The images obtained from the FORTE satellite include parts of the satellite. i.e., the long antenna and a thin aiming cross. Those parts are present in a stationary way, making them easier to identify [1]. DIP techniques are used to solve a variety of problems. Geographers use the same or similarly technique to study pollution pattern from aerial and satellite imageries [2]. Image processing is often a pre-processing stage preceding Pattern Recognition, in order to provide patterns, which are effective and efficient measurements of an image. Image processing means analysis of the image that is extracting the features. We must classify the images. Pattern Recognition does this classification and description. Conversely, PR can be a mainstream processing produced in IP. In order to determine a good set of characteristic measurements and their relationship for the representation of the objects. PR is sometimes needed to classify or cluster primitives or measurement of the images. The data analysis can be separate procedure linking DIP and PR. It can be a part of IP or a part of PR. Therefore, in object, or pattern recognition the general procedures are IP, Data analysis and PR [3].

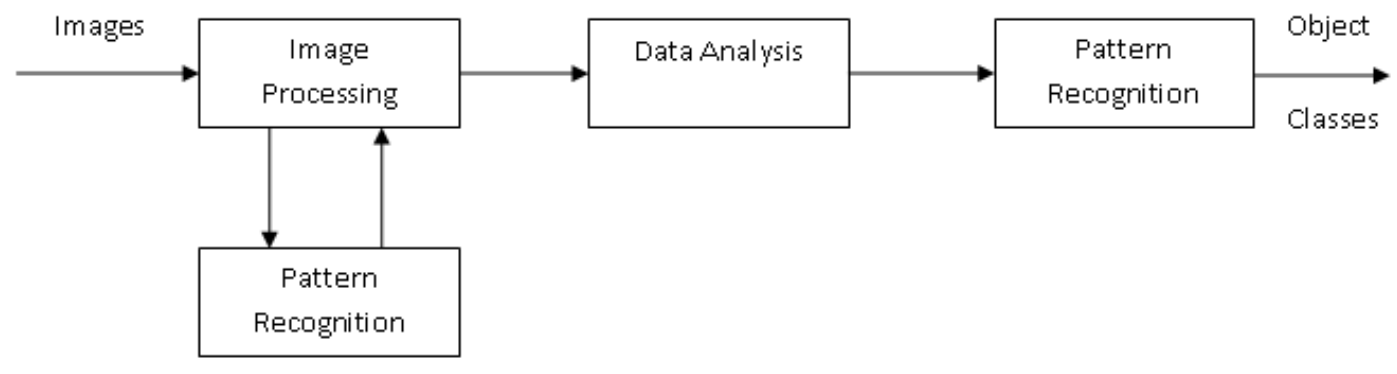

Figure 1.1. Diagram of Object Recognition [Source: as in [3]]. 


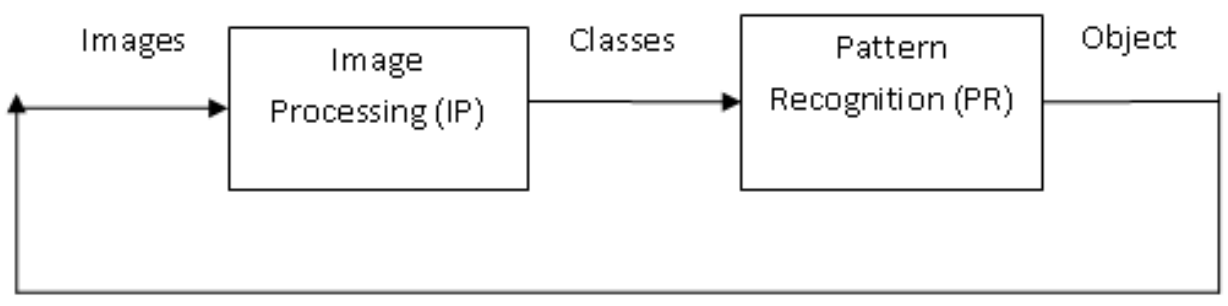

Figure 1.2 Relations Between PR and IP[Source: as in [3]].

The key concept to be kept in mind is that selecting the descriptors on which to base each component of a pattern vector has a profound influence on the eventual performance of object recognition based on the pattern vector approach. Pattern characteristics are best described by structural relationships. Hence not only quantitative measures about each feature but also the spatial relationships between the features and they are best solved by structured approaches. Indirectly usage statistical means using probability statistic techniques.

1.3 Spatial Techniques: One of the simplest approaches for detecting changes between two image frames $\mathrm{f}(\mathrm{x}, \mathrm{y}, \mathrm{ti})$ and $\mathrm{f}(\mathrm{x}, \mathrm{y}, \mathrm{tj})$ taken at time ti and tj respectively is to compare the two images pixel by pixel [3]. One procedure for doing this is to form a difference image. Suppose that we have a reference image containing only stationary components. Comparing this image against a subsequent image of the same scene, but including a moving object, results in the difference of the two images cancelling the stationery elements, leaving only nonzero entries that correspond to the non-stationery image components.

\section{PROBLEM STATEMENT}

The movement of the cloud is arbitrary in nature the identification of clouds as segments in the image has to use the techniques derived from pattern recognition or rules based on fuzzy logic. It is a problem of both mid level image processing, some concepts of high level image processing except computer vision, and knowledge base. A mid level process is characterized by the fact that its input are images, but its outputs are attributes extracted from the images (E.g., the identity of individual objects) and higher-level processing involves " making sense" of an ensemble of recognized objects. This involves Declarative knowledge base method, which is based on some decisions. Knowledge about the problem domain is coded into an image processing system in the form of the knowledge database. This knowledge may be as simple as detailing regions of an image where the information of interest is known to be located. Thus limiting the search that has to be conducted in seeking that information. In addition to guiding the operation of each processing module, the knowledge base also controls between processing modules. For example: let us consider the ozone layer in which the holes are to be detected. For this, high-level knowledge would be the continuity of the concentration figures and the fact that areas of similar concentration appear as annuli centered at the polar area.

\section{MOTIVATION}

From the beginning of science, visual observation has played a major role. To capitalize on this talent by providing satellite image data in a format that can be interpreted to gain new insight about earth. The first problem is that the satellite data collected by government agencies are in digital format. How to convert the brightness values is the creation of brightness map also 
commonly refers to as gray scale image. Satellite images are compact and very sensitive to distortion. A new generation of polar orbiting meteorological satellites was initiated with the launch of the TIROS-N satellite. The SR was replaced with the advanced Very High Resolution Radiometer (AVHRR), which yields images in four channels. The additional channels provide a potential for extracting more information than can be extracted from two channels. With the growing interest in developing cloud climatology, there are now a number of techniques for extracting cloud cover parameters from the satellite measurements [4]. Automatic methods are primarily used for cartography of the cloud-top level, for example in ESOC (European Space Operation Center). For cloud classification itself, methods using typical classes defined by meteorologists have been tried [6]. Researchers have mentioned that the identification of cloud and its movement has various complex methods because of irregular shapes and arbitrary movement. This motivation of challenging work has led to the journey of proposed work. INSAT spacecrafts are capable of providing the imageries at every half hour intervals in case of urgent need. Nominally about 15 times in a day these imageries are obtained from a Satellite by India Meteorological department. In case of any adverse weather system development, like cyclone, additional imageries are taken at more frequent intervals. The present image Data Acquisition system is used only for Quick look health monitoring of the instrument that provides the image data. Hence the value addition through this proposal software solution will enhance the system's role in effective utilization of image data collected from Spacecraft.

\section{RELATED RESEARCH}

A technique is described for extracting cloud cover parameters from multispectral satellite radiometric measurements. Utilizing three channels from the AVHRR on NOAA polar orbiting satellites, it is shown that one can retrieve four parameters from each pixel: cloud fraction within the FOV, optical thickness, and cloud-top temperature and a microphysical model parameter [4]. The attempt to distinguish land from water in satellite images, specifically image is taken by the FORTE satellite. First approximate areas hidden by stationery artifacts in the image is found out and then segment the regions of land from water, finally the boundaries of the surrounding landmasses is determined [1]. A common way to represent shape is based on so-called key points and leads to shape variables, which are invariant with respect to similarly transformations [5]. To find out the practical position estimation system of an aircraft using sequential aerial images. Relative position estimation recursively computes the current position of an aircraft by accumulating relative displacement estimates extracted from two successive aerial images. Simple accumulation of parameter values decreases the reliability of the extracted parameter estimates as an aircraft goes on navigating, resulting in a large position error [2]. A system for automatically identifying the script used in a handwritten document image is described. The system was developed using a 496-document dataset representing six scripts, eight languages, and 279 writers. Documents were characterized by the mean, standard deviation, and skew of five connected component features. A linear discriminate analysis was used to classify new documents, and tested using writersensitive cross-validation. Classification accuracy averaged $88 \%$ across the six scripts. The same method, applied within the Roman subcorpus, discriminated English and German documents with $85 \%$ accuracy[8]. Multi-layered image-based rendering system that can use different types of input to produce and render new environments. The ideas embodied in the multi-layered IBR system are useful in augmenting the capabilities of applications that require fast and geometrically consistent rendering of 3-D scenes such as video editing [9]. The recognition of abdominal organs is established based on a contextual neural network, fuzzy rules. The contextual neural networks are used to label the image pixels into disjointed regions, followed by a fuzzy rule based recognition 
system to identify organs from these regions. Because of the proper usage of the overlapping information, separated regions of a same organ, such as a the left and right lobes of the liver can be successfully recognized [10].

\section{IDENTIFICATION OF THE MOVEMENT OF THE CLOUDS}

In this paper it is an attempt to achieve the goal of early warning system. The paper will explains how to identify the region of clouds, extent of the cloud and the proximity of the cloud cover to the landmark of interest. Since the cloud formation is arbitrary in nature the identification of clouds and its manipulation is very important to satisfy the purpose. Identifying cloud regimes and their role in the climate system can serve a multitude of purposes, ranging from a better understanding of clouds to guiding field experiments to improving the representation of clouds in models [7]. To find the centroid for the region of interest this has to be segmented from the satellite image. Using the centroid of the reference image and the next successive image one can found distance between the regions. These points are considered as reference centroid and distance of the reference image.

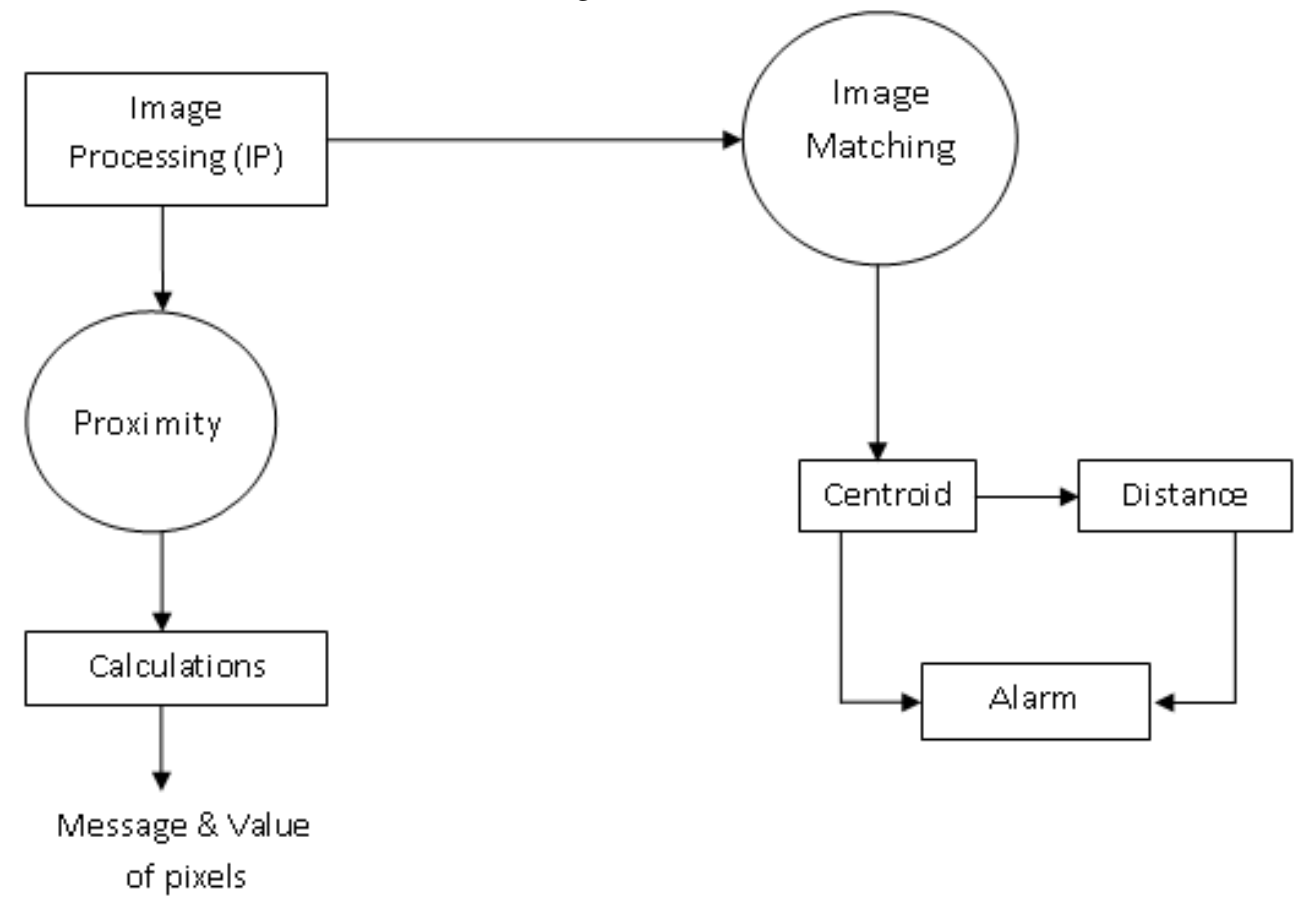

Figure 2: Algorithm for Identification of Movements of Clouds

For our convenience region $\mathrm{A}$ is assumed as earth region and region $\mathrm{B}$ is assumed as cloud region

\section{a. Method for computing the Centroid:}

Take regions of segmented area by applying the formula for centroid.

Centroid C $=($ Value of rows/ Number of Pixels, Value of columns / No. of pixels)...... (Eqn 1)

Algorithm:

Input: Segmented region(image): two dimensional region which contains gray values of the image.

Output: $\mathrm{C}$, Centoid which is numeric

Method: Compute Centroid C = (Value of rows/ Number of Pixels, Value of columns / No. of pixels) 


\section{b. Method for computing the Distance:}

Once we get the centroid of two regions from the above algorithm i.e. (x1, y1) and (x2, y2), we can easily find the distance by using distance formula.

Distance $\mathrm{D}=$ Square root of $\left(\left(\mathrm{x}_{2}-\mathrm{x}_{1}\right)^{\wedge} 2,\left(\mathrm{y}_{2}-\mathrm{y}_{1}\right)^{\wedge} 2\right)$

Algorithm:

Input: $\left(\mathrm{x}_{1}, \mathrm{y}_{1}\right) \&\left(\mathrm{x}_{2}, \mathrm{y}_{2}\right)$

- Centroids of two regions

Output: D, distance between two regions of the reference image.

Method:

$$
\text { Compute } \mathrm{D}=\operatorname{Sqrt}\left(\left(\mathrm{x}_{2}-\mathrm{x}_{1}\right)^{\wedge} 2,\left(\mathrm{y}_{2}-\mathrm{y}_{1}\right)^{\wedge} 2\right)
$$

Now consider centroid $\left(\mathrm{x}_{1}, \mathrm{y}_{1}\right)$ and $\left(\mathrm{x}_{2}, \mathrm{y}_{2}\right)$ and distance $\mathrm{D}$ as reference.

\section{c. Method for estimating the model of the cloud:}

We look for next successive image and grid remain constant. We will get different image values for different images. Let the next successive image be named as region B1. The processing involves the following steps:

i. Image matching: The values are stored in a database separately for the different images of cloud region.

- Subtract the two images and store the resultant image.

- Find the centroid of resultant image.

- Find the shift $\left(\mathrm{x}_{211}, \mathrm{y}_{211}\right)$

- Find the distance $\left(D_{1}\right)$ between the new cloud region and reference earth region using distance algorithm.

ii. Proximity of the Cloud: the percentage of the cloud above the earth region determines the proximity of the cloud.

- Count the number of pixels of cloud region.

- Find the number of pixels acquired by the land region.

- Find the mean and fix the threshold value.

- By comparing with the threshold value you will get the thickness of the cloud.

iii. Scaling: By increasing the $\mathrm{x}$ axis and $\mathrm{y}$ axis by one pixel each we can take bigger part of the region.

d. Alarm: This is the major discussion of this paper.

Input: $\mathrm{D}$, distance between the earth and cloud region of the reference image.

D1, new distance between the earth region of reference image and cloud region of new image.

$\left(\mathrm{x}_{2}, \mathrm{y}_{2}\right)$, centroid of reference cloud region.

$\left(\mathrm{x}_{211}, \mathrm{y}_{211}\right)$, centroid of the resultant image of the difference.

Output: $\mathrm{D}_{3}$, output distance, message or warning.

Method:

- Compare the distances in the two images

- Now the decision making is based on this criterion.

- If the resultant distance is lesser than the difference between distance between the earth and cloud region of the reference image and the new distance between the earth region of reference image and cloud region of new image (refer eqn 2) then message will come as "Cloud is coming nearer to earth" otherwise message "cloud is going away from the earth" will appear on the screen.

- If y211 is less than y2 and the difference between $x 2$ and $x 211$ is less than epsilon (e) then message/warning "Cloud is accumulating and movement is anticlockwise" will 
appear otherwise check for alternative decision and say "Cloud is accumulating and movement is clockwise" else message "Cloud is dispersing" will appear on the screen.

e. Database Design: for storing purpose, a reference image is displayed using display algorithm and corresponding pixel values are stored in the data files (.DAT). After segmentation of the reference image. 2 regions are available and the corresponding pixel values of two regions are stored in another two data file. The two data files are used for further processing. It can be processed using different kinds of data structures like single dimension array or two dimensional array or files. Using the third data file resultant matrix can be stored and later this can be used to get the resultant image.

\section{CONCLUSION}

The goal of early warning plays a very important role. For this identification of movement of cloud is very essential. The findings of the centroid, distance, proximity of cloud, image matching, and movement of clouds helps in developing warning or message about the status of the cloud. This kind of warning or message will help in the areas where the occurrence of cyclone is arbitrary and it can be used for predicting the rainfall or cyclone formation. Dependencies on the occupancies on the value of the region we should be in the position to predict that the next successive image how the patterns are moving. Pattern is changing. Due to this the subsequent future work will be to interconnect the regions of interest using higher domain knowledge and other expert system tools. In the proposed paper database is required for cataloguing, storing, retrieving and annotating the set of satellite images. This plays a very important role in processing, comparing and help in taking decisions. The important concept of designing a database is a great deal of programming time is saved because the RDBMS handles the construction of data. The RDBMS is the most complex varieties of software in existence. One way to get a feel for the different aspects of a DBMS is, considering the various kinds of users of such a system and their ways. They interact with the system and each others. For the purpose of implementation the satellite images were taken every 1-hour of time interval.

\section{REFERENCES:}

1. Karina Waldemark, Thomas Lindblad, Vlatko Becanovic, Jose L.L. Guillen, Philip L. Klingner, (2000). "Patterns from the sky Satellite image analysis using pulse coupled neural networks for pre-processing, segmentation and edge detection", Pattern recognition letters 21 (2000) 227-237.

2. Dong-Gyu Sim, rae-Hong Park, Senior Member, IEEE, Rin-Chul Kim, Member, Ieee, Sang Uk Lee, senior Member, IEEE, and Ihn-Cheoi Kim, (2002) "Integrated Position Estimation Using Aerial Image Sequences", IEEE transactions on Pattern Analysis and Machine Intelligence, Vol 24, No. 1, January 2002.

3. Rafael C. Gonzalez, Richard E. Woods. (2000). "Digital Image Processing". $5^{\text {th }}$ edition, pearson Education.

4. Albert Arking and Jeffrey D. Childs, (1984) "Retrieval of Cloud Cover Parameters from Multispectral Satellite Images", published in journal of Climate and Applied Meteorology. Vol 24, 11 december 1984.

5. Anke Neumann, (2003) "Graphical Gaussian Shape Models and Their Application to Image Segmentation", IEEE transactions on Pattern Analysis and Machine Intelligence. Vol 25, No.3, March 2003. 
6. Michel Desbois, Genevieve Seze and Gerard Szejwach, (1982) "Automatic Classification of Clouds in METEOSAT Imagery: Applications to High-Level Clouds".

7. Christian Jakob, George Tselioudis. (2003) "Objective identification of cloud "regimes in the Tropical Western Pacific". Published 1 November.

8. Judith Hochberg, Kevin Bowers, Michael Canno and Patrick Kelly" Script and language identification for handwritten document images", International Journal on Document Analysis and Recognition, 1433-2833 (Print) 1433-2825 (Online), Vol 2/numbers 2-3, December, 1999.

9. Sing Bing Kang, Huong Quynh Dinh, "Multi-layered image-base rendering", research publications of Microsoft.

10. Chien-cheng lee, Pau-choochung and Hong-Ming Tsai, "Automatic recognition and identification of abdominal organs in CT images", international computer symposium a workshop on image processing and character recognition, December 17-19, 1998, NCKU, Taiwan.

\section{TEXT BOOK REFERENCES}

1. Bernd Jahne, (1997),Digital Image Processing, $5^{\text {th }}$ edition, Springer-verlag.

2. Julio Sanchez and Maria P Canton, (1999), Space Image processing, CRC press published by Library of congress cataloguing-in-publishing-data.

3. M.A. Sid Ahmed, (1994), Image Processing, McGraw Hill publication

4. G.J Awcock and R. Thomas, (1995), Applied image processing, McGraw Hill, Inc

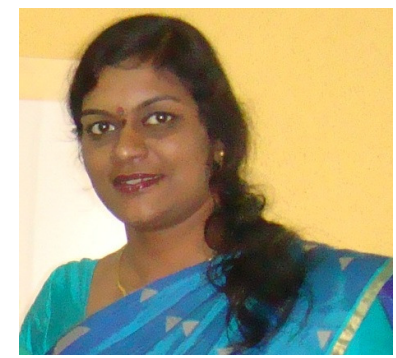

\section{AUTHOR's BIOGRAPHY}

Ms. Ramya S Gowda is working as consultant in Manipal Universal Learning Pvt Ltd and Lecturer in Information Systems for the Department of Management and Commerce of Directorate of Distance Education, Sikkim Manipal University. She has done MS (int) Computer Science and Engineering from the University of Mysore, Manasagangothri, MYSORE. She has 4.5 years of experience which includes Teaching and Research. She was working as Scientist ' $\mathrm{C}$ for 6 months for the Department of Space, Master Control Facility (MCF), ISRO, Hassan. She was also doing her research on 'Automatic Classification of Satellite Images for Weather Monitoring'. She has 
written around research papers that are published in National and International conferences. She has presented her paper in various national and international conferences. Being an Academician Her subject of interest varies from Science and Technology to Information Technology which includes Digital Image Processing, System Software, Database Management Systems, Data warehousing and Mining, ECommerce, ERP, Simulation and Modeling, Object Oriented Analysis and Design, Analysis and Design of Algorithms and many more... 